\title{
THE NEUTRALIZATION OF SPACE CHARGE BY POSITIVE IONS IN CAESIUM VAPOR
}

\author{
By V. E. Whitman
}

\section{ABSTRACT}

A tube was constructed in which the neutralization of space charge by positive ions was studied. The positive ions were formed by the photo-electric ionization of caesium vapor. The change in current due to the illumination was measured as a function of such controllable variables as pressure, light intensity, anode voltage, and cathode emission. An empirical relation between change in anode current and illumination is given which may be fitted to the experimental results by adjusting one constant.

CONTENTS

I. Introduction

1. Historical

2. Preliminary measurements

II. Apparatus

III. Methods and results.

IV. Discussion

V. Conclusions

VI. Acknowledgments

\section{INTRODUCTION}

\section{HISTORICAL}

The maximum current which can be carried by ions or electrons between electrodes is determined by the form of the electrodes, the potential difference between them, the mass or the ion or electron, and the charge it carries. The flow of current is said to be limited by space charge.

From the viewpoint of theory and experiment taken together, the geometrical arrangement which lends itself best to investigation is that of concentric cylinders with the inner as cathode. Such an arrangement is usually sealed in a highly exhausted inclosure. The cathode, in general, consists of a wire which is heated by the passage of an electric current and thus made to emit electrons.

In high vacuum thermionic devices of the type in which the anode is a flat plate, the few positive ions formed play an insignificant part in the conduction. Under ordinary conditions they move over to the cathode and discharge or else move out from the field between the electrodes. In neither case do they contribute appreciably to the neutralization of the electron space charge.

If, however, the thermionic device is made so that the cathode lies along the axis of a cylindrical anode with closed ends except where the cathode leads must penetrate any positive ion which is formed within the inclosure, having a component of velocity transverse to the radius of the cylinder, will, in general, not fall directly into the cathode, but 
will perform some type of orbit within the field. Moreover, the closed end construction referred to will greatly reduce the chances of the ion wandering from the inclosure. Such an imprisoned ion will execute orbits to-and-fro across the tube as long as it has any component of velocity transverse to the radius. This necessary transverse velocity is present in the thermal agitation of the atoms.

The more excursions a positive ion makes across the tube before it strikes the cathode, the more effective it is in neutralizing the space charge. The condition that a positive ion starting from a point immediately inside of the anode shall just fail to strike the cathode is that $V$, the potential difference between anode and cathode shall be less than the value given by

$$
\text { e } V=\frac{1}{2} m v_{0}^{2}\left(\frac{R}{r}\right)^{2}
$$

In this equation, as given by Hull ${ }^{1} m$ is the mass and $e$ the charge on the ion, $v_{o}$ the initial transverse velocity, and $R$ and $r$ the radius of anode and cathode, respectively.

Within the range of conditions for which equation (1) is valid we see that (1) for given $V$ and $\frac{R}{r}$ the proportion of ions which will fail to reach the cathode on their first trip is the same for all gases at the same temperature; and (2) the higher the temperature of the gas in the tube, the greater will be the average value of $\frac{1}{2} m v_{o}^{2}$ and the greater will be the proportion of ions missing the cathode.

Kingdon ${ }^{2}$ used a tube in which a cylindrical anode practically surrounded the electron-emitting portion of a concentric hot wire cathode, and worked with $\mathrm{He}, \mathrm{H}, \mathrm{Ne}$, and $\mathrm{Hg}$ at pressures ranging from $10^{-2}$ to $10^{-7} \mathrm{~mm}$. The effectiveness of a positive ion in neutralizing the space charge was found to vary as the $2 / 3$ power of $\frac{1}{p}$. Over the range of pressures used, the results were consistent with the assumption that the recombination of positive ions with electrons was negligible.

Now the contribution to the space charge by a particle carrying a charge is proportional to the time $\tau$ consumed in making the trip between the electrodes once times the number of trips. Approximate calculations showed that in one of the tubes used by Kingdon with helium $\left(\tau_{+}\right)$for the ion was about $1.4 \times 10^{-6}$ secs. and $\left(\tau_{-}\right)$for the electron was about $1.08 \times 10^{-8}$ seconds for $V=35$ volts. Hence, one ion moving once from anode to cathode will neutralize the space charge of $\tau_{+} / \tau_{-}=130$ electrons. This number of electrons in excess of the normal space-charge current will then flow across the tube. A calculation led Kingdon to believe that in $\mathrm{He}$ at $10^{-5} \mathrm{~mm}$, an ion which just missed the filament on its first passage across the tube might travel across the tube as many as 350 times before losing its energy and discharging to the cathode. It is thus conceivable that one helium atom might neutralize the space charge effect of as many as $4.5 \times 10^{4}$ electrons in the type of tube used by Kingdon. 
Foote and Mohler ${ }^{3}$ utilized this effect for measuring the photoelectric ionization of caesium vapor. The form of tube used was similar to that shown in Figure 1 except that no electrical shielding was provided. The left-hand end of the tube was fitted with a plane quartz window through which light of various wave lengths was passed into the anode cylinder. A gauze at the left-hand end of the anode cylinder served to effectively imprison the positive ions formed. The filament was run at a temperature high enough so that the current to the anode would be limited by space charge, but sufficiently low so that the filament would not emit positive ions.

Under these conditions, current-voltage characteristics of the tube were obtained when the space between anode and cathode was illuminated with light of various wave lengths. Over the range of vapor pressures covered, the change in thermionic current on illumination was found to increase with the pressure, as shown in Table 1.

TABLE 1.-Change in current due to radiation as a function of vapor pressure

\begin{tabular}{|c|c|c|}
\hline Temperature, $^{\circ} \mathrm{C}$. & $\begin{array}{c}\text { Pressure in } \\
\mathrm{mm} \mathrm{Hg}\end{array}$ & $\Delta_{i}$ \\
\hline 135 & $\begin{array}{l}0.00048 \\
.001125 \\
.0073 \\
.0090 \\
.0313\end{array}$ & $\begin{array}{l}\text { Amps. } \\
0.7 \times 10-5 \\
1.1 \times 10-5 \\
2.0 \times 10-5 \\
2.2 \times 10-5 \\
3.2 \times 10-5\end{array}$ \\
\hline
\end{tabular}

The vapor pressures were calculated from the temperature measurements using the work of Langmiur and Kingdon. ${ }^{4}$

The increase in thermionic current due to illumination was found to increase with larger filament emission.

The change in current with radiation was found to be nearly independent of voltage below the ionization potential, but to approach zero rapidly when ionization by electron impact occurred.

This work was continued in a second paper by Mohler, Foote, and Chenault, ${ }^{5}$ in which the relative photosensitivity - that is, the ions per unit intensity-was investigated as a function of the wave length.

Throughout this work it was assumed, and experimentally verified, that the change in current due to radiation was directly proportional to the intensity of the radiation. This was over a relatively low range of intensities. It is evident that if the intensity were increased indefinitely, the effect of space charge would finally be completely wiped out, after which a further increase in intensity would have no effect on the current. The theoretical aspects of this nonlinear relation at high intensities become too complicated to allow much analytical investigation of the phenomenon.

In some unpublished experiments Mohler used a beam of electrons as the ionizing source. He found that particularly at low pressures, and over a limited range of ion currents, fairly reliable values could be gotten of the absolute number of electrons released from the cathode region by one positive ion.

3 Phys. Rev., 26, 195; 1925. $\quad{ }^{4}$ Proc. Roy. Soc., 107A, p. 61; 1925. $\quad$ s Phys. Rev., 27, p. 37; 1926.

$84789^{\circ}-29-11$ 


\section{PRELIMINARY MEASUREMENTS}

Because of the incompleteness of the available experimental data it was thought desirable to irvestigate the phenomenon of the neutralization of space charge by positive ions, throughout a greater range of experimental conditions than had heretofore been recorded. Some differences should be noted between the conditions under which Kingdon's ${ }^{6}$ experiments and those described here were performed. In the present work there were probably no true ion orbits as analyzed by Kingdon. In the experiments to be described, higher pressures and lower voltages were used than by Kingdon. It was decided to use radiation rather than electrons as the source of ionization because of the greater reliability of the results over the range of variables investigated. The use of radition has one disadvantage, namely, that there is no way approaching reliability with which the absolute number of ions present can be calculated. A rough estimate may be obtained from some recent absolute measurements of photoionization to be published shortly by Mohler and Boeckner. From this work it appears that the ion current is of the order of $10^{-4}$ times the change in electron current.

$\mathrm{X}$ rays from a molybdenum tube of the Collidge type were first tried as the ionizing source. The X-ray tube was run at as near constant output as could be gotten from the power circuits available. The tube in which the space charge was produced and neutralized was caesium filled, and in all respects similar to that used by Foote and Mohler. ${ }^{7}$ All other variables were held constant and the change in current due to illumination was measured as a function of the vapor pressure of the Cs. As predicted by Kingdon's theory, the effect at first increased with increasing pressure. With 33,000 r. m. s. volts across the X-ray tube, and a current consumption of 15 milliamperes, the change in current in the caesium tube due to illumination with the $\mathrm{X}$ rays became a maximum at a vapor pressure of caesium of 0.43 $\mathrm{mm}$. of mercury. Above this pressure, the change was less.

These experiments were sufficient to prove that the effect with $X$ rays was real, but small for the intensities of $X$ rays available. Difficulty was also experienced in shielding the galvanometer system from electrical disturbances arising in the high tension X-ray tube circuit.

Light from a concentrated filament lamp was chosen as the source of ionization in the subsequent experiments because of the high intensities available, and because of the ease with which its intensities could be controlled and reproduced. The original caesium tube had to be abandoned because it contained a relatively low resistance electrical leak between anode and cathode.

\section{APPARATUS}

A tube of the design shown in Figure 1 was made. This was not essentially different from that originally used except for the addition of the guard ring shown. 
The window at the left-hand end of the tube in Figure 1 was a plane piece of clear fused quartz. The anode cylinder was made of platinum and measured approximately $3 \mathrm{~cm}$ diameter by $10 \mathrm{~cm}$ length. The filament was of 5-mil tungsten wire, oxidized before inserting the caesium. The usual precautions of high temperature prolonged baking with pumps running were observed before the caesium was finally distilled into the tube and the tube sealed.

The electrical circuits associated with this tube are also to be seen in Figure 1. By means of the potentiometer arrangement shown at $E_{3}$ it was possible to read changes in anode current of the order of $10^{-8}$ amperes on the galvanometer, while the total anode current might be of the order of $10^{-3}$ amperes.

A 400 -watt lamp of the type used in motion-picture projectors was used as the source of light. A quartz lens was used to render the light passing into the tube approximately parallel. The intensity of the light entering the tube was controlled by inserting one or more nickel gauze screens in the path of the light. Without screens, the light

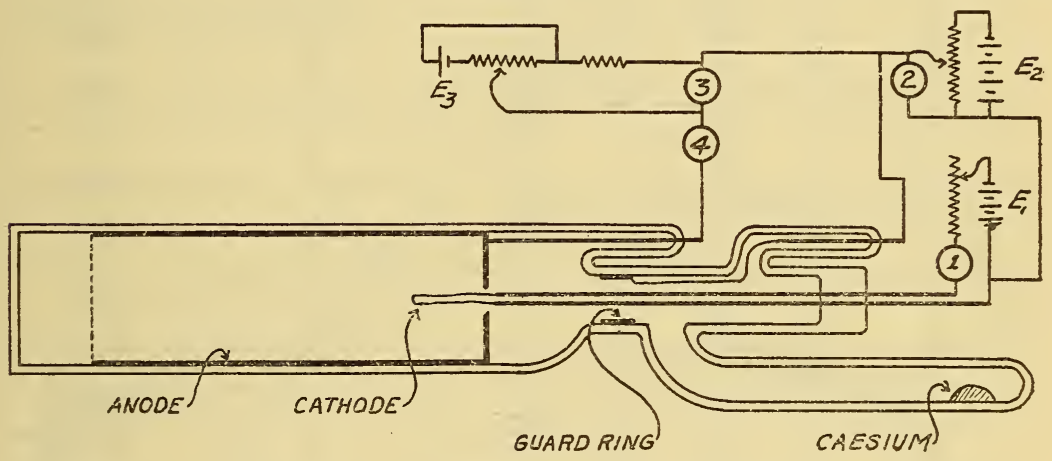

FigURe 1.-Tube and associated apparatus

$E_{1}, E_{2}, E_{3}$, Batteries.

(1) Ammeter. (2) Ammeter.

(3) Galvanometer. (4) Milliammeter.

intensity at the tube was found to be 1,600-foot candles. Each screen was found to have a transmission of 0.25 .

The main body of the tube shown was maintained at a temperature not greatly different from $300^{\circ} \mathrm{C}$. The temperature of the side tube containing caesium was varied to adjust the vapor pressure. These pressures were calculated from the temperature of the side tube, using the curve selected by Little. ${ }^{8}$

\section{METHOD AND RESULTS}

Before making any electrical measurements at any pressure, the temperature of the tube was held constant for several hours so that equilibrium might be reached. For the low pressures; that is, low temperatures, as many as 15 hours were allowed for reaching this equilibrium. For the highest pressures only 3 hours were allowed.

Some uncertainty was felt as to just how the filament current should be set so that readings under otherwise different conditions might be 
comparable. Check readings could not be obtained when the same filament current was used at the same vapor pressure of caesium on consecutive days. This was due to the changing emission characteristics of the filament as a result of prolonged high temperature exposure to caesium vapor. The method adopted for setting the filament current was as follows: After pressure equilibrium had been reached, 10 volts were applied between anode and cathode and the filament current increased until the anode current was 1 milliampere. In the single case of the upper curve of Figure 6, the filament temperature was obtained by setting for 15 milliamperes at 10 volts.

With a given filament setting, readings were made of the change in anode current as a function of light intensity for several voltages below the ionization potential of caesium vapor.

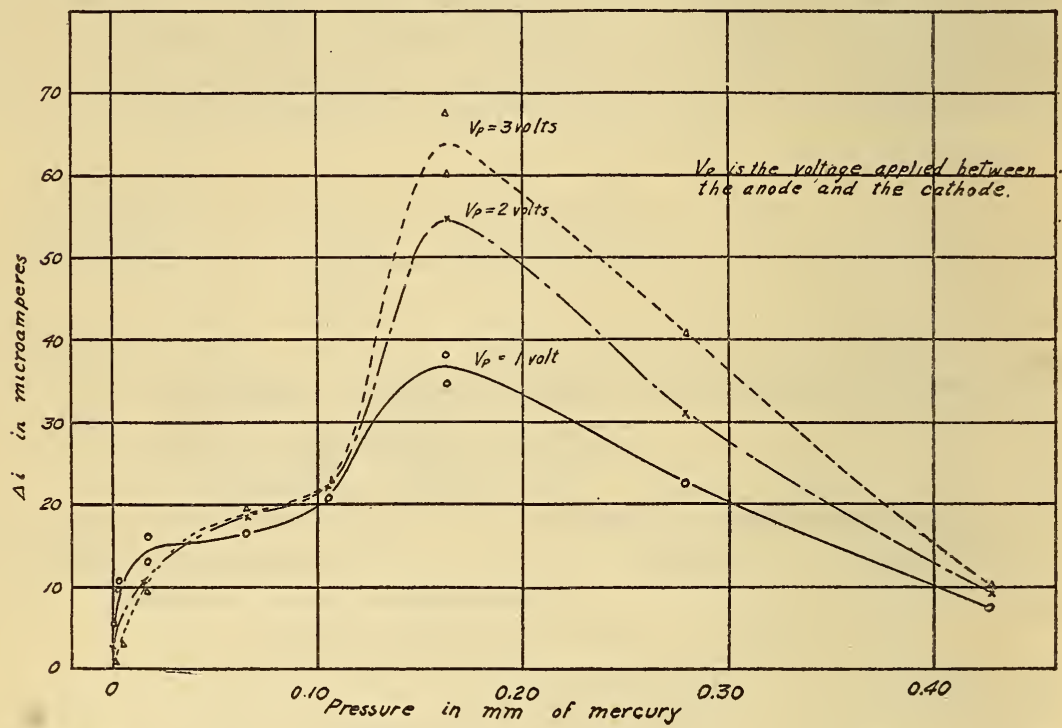

FIgURE 2.-Variation of $\Delta i$, at maximum illumination, with pressure for three different anode voltages

Preliminary runs showed that the photo-electric effect on the electrodes was negligible; also that the magnetic field set up by the electric heating furnaces had a negligible effect.

Table 2, together with the curves referred to in its last column, give the results of these measurements of $\Delta i$, the change in anode current due to illumination with light of intensity $I$. The table only gives values of $\Delta i$ for the same maximum illumination, taken as unity in all cases. The points on the curves listed in the right-hand column are for other values of light intensity. Figure 2 showing $\Delta i$ at various voltages as a function of pressure was plotted from the data contained in Table 2 and its associated curves. 
TABLE 2

\begin{tabular}{|c|c|c|c|c|c|}
\hline Pressure of $\mathrm{Cs}$ in $\mathrm{mm} \mathrm{Hg}$ & $\begin{array}{c}\text { Anode volt- } \\
\text { age }\end{array}$ & $\begin{array}{l}\text { Anode cur- } \\
\text { rent in mi- } \\
\text { croamperes }\end{array}$ & $\begin{array}{c}\Delta_{i} \text { in micro- } \\
\text { amperes for } \\
I=1.00\end{array}$ & $K_{3} \times 10^{-0}$ & $\begin{array}{l}\text { Curve in } \\
\text { figure }\end{array}$ \\
\hline $\begin{array}{l}0018 \\
0039 \\
0039 \\
0185 \\
0185 \\
066 \\
066 \\
164\end{array}$ & $\begin{array}{l}1.0 \\
1.0 \\
3.0 \\
1.0 \\
3.0 \\
3.0 \\
1.0 \\
3.0 \\
1.0 \\
3.0 \\
1.0 \\
3.0\end{array}$ & $\begin{array}{r}14.3 \\
10.9 \\
76.0 \\
6.5 \\
41.4 \\
55.8 \\
2.1 \\
14.0 \\
3.0 \\
10.6 \\
15.9 \\
41.2\end{array}$ & $\begin{array}{r}5.53 \\
10.87 \\
2.69 \\
16.14 \\
11.68 \\
45.70 \\
16.20 \\
19.50 \\
34.6 \\
63.1 \\
7.42 \\
9.83\end{array}$ & $\begin{array}{l}9.1 \\
4.2 \\
4.7 \\
2.74 \\
1.62 \\
.22 \\
3.4 \\
1.53 \\
.77 \\
.22 \\
5.8 \\
2.0\end{array}$ & $\begin{array}{r}4 \\
5 \\
5 \\
6 \\
6 \\
6 \\
7 \\
7 \\
8 \\
8 \\
\end{array}$ \\
\hline
\end{tabular}

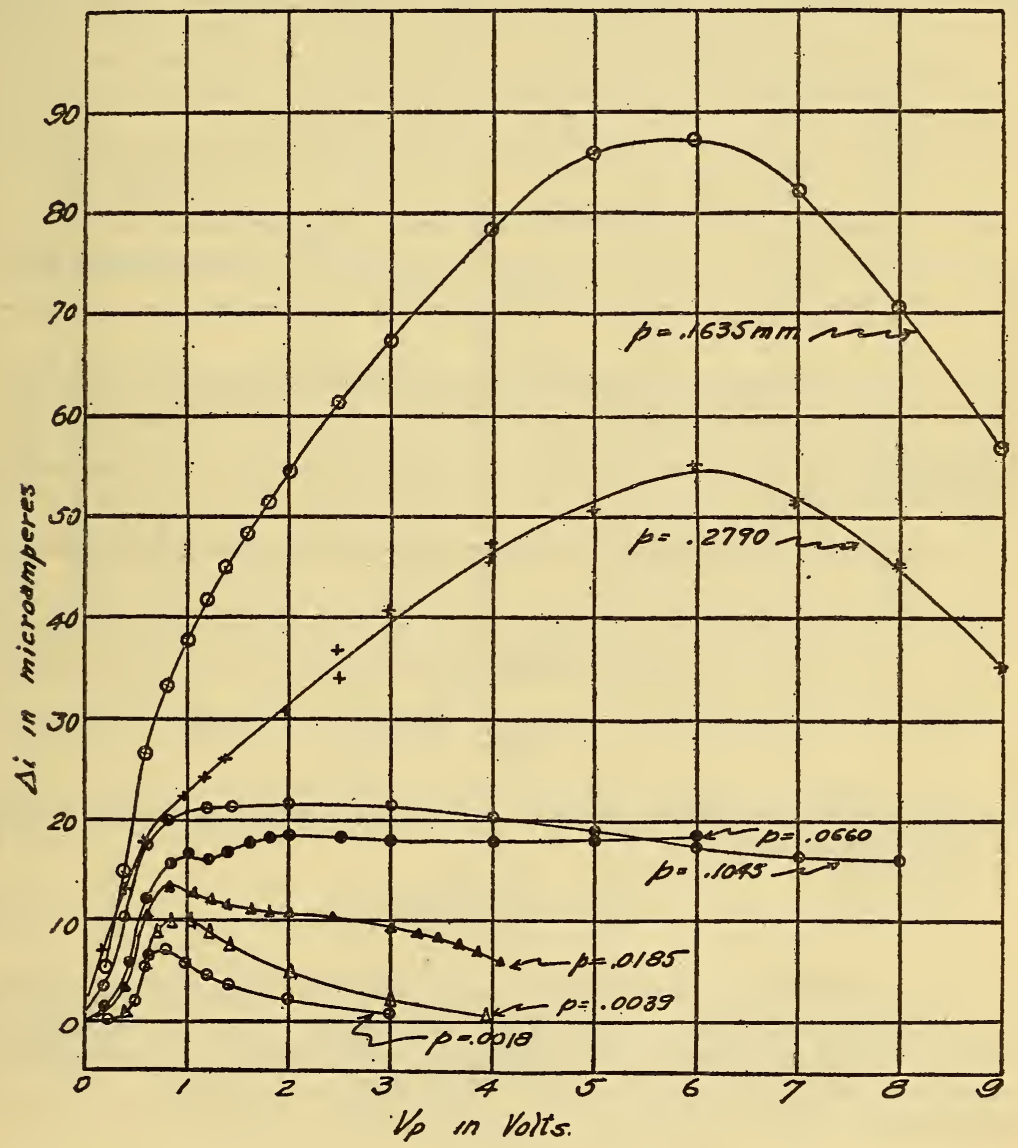

FIgURE 3.-Variation of $\Delta i$, at maximum illumination, with anode voltage, at different pressures

Using the full intensity of illumination, the change in anode current was investigated as a function of the anode voltage at various pressures. The results of these measurements are given in the curves of Figure 3. 


\section{DISCUSSION}

A rigorous analytical attack of the problem of the neutralization of space charge by positive ions would involve difficulties out of proportion to the usefulness of the solution if one were possible. It is desirable, however, to present an empirical relation which fits the results.

Let it be assumed that the rate of production of ions is proportional to the intensity $I$ of the light, and that $\tau$ is the average life of an ion. Then the number of ions present will be $a I \tau$.

Second, let it be assumed that the change in anode current $\Delta i$ is proportional to the number of ions present; that is, $\Delta i=b a I \tau$.

If it were assumed that the loss of ions is proportional to the conconcentration, then $\Delta i$ would be proportional to the intensity $I$. This is not observed. The addition of a term involving a loss of ions

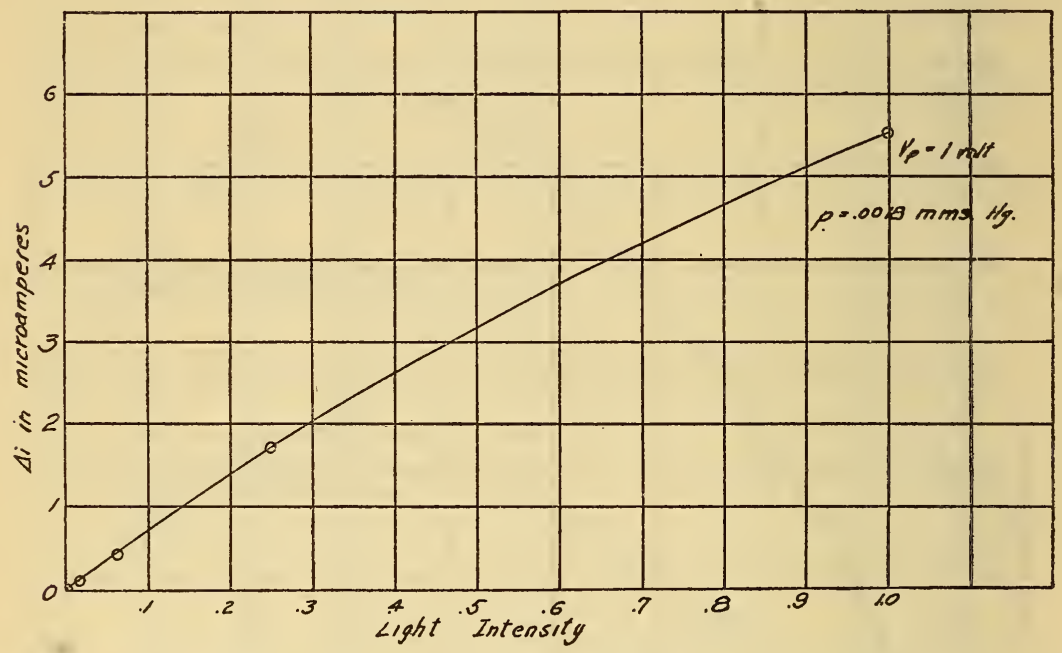

FIGURE 4.-Variation of $\Delta i$, with light intensity for conditions noted on curves

proportional to the total current $(i+\Delta i)$ gives an equation of the form of equation (2)

$$
\Delta i=\frac{b a I}{c+d(i+\Delta i)}
$$

which can be fitted to the present experimental data by the suitable choice of two arbitrary constants. However, $c$ of equation (2), which comes in on account of a diffusion assumption, is negligible in comparison with the product $a b$. This allows equation (2) to be simplified to

$$
\frac{I}{\Delta i}=K_{3}(i+\Delta i)
$$

which fits the data within the experimental error, and with the use of only one arbitrary constant. 
Figures 4, 5, 6, 7, and 8 are intended to show the agreement between the experiments and equation (3). The points indicated along the curves were experimental determinations. For each condition $K_{3}$ was calculated from the value of $\Delta i$ at $I=1.0$. This was con-

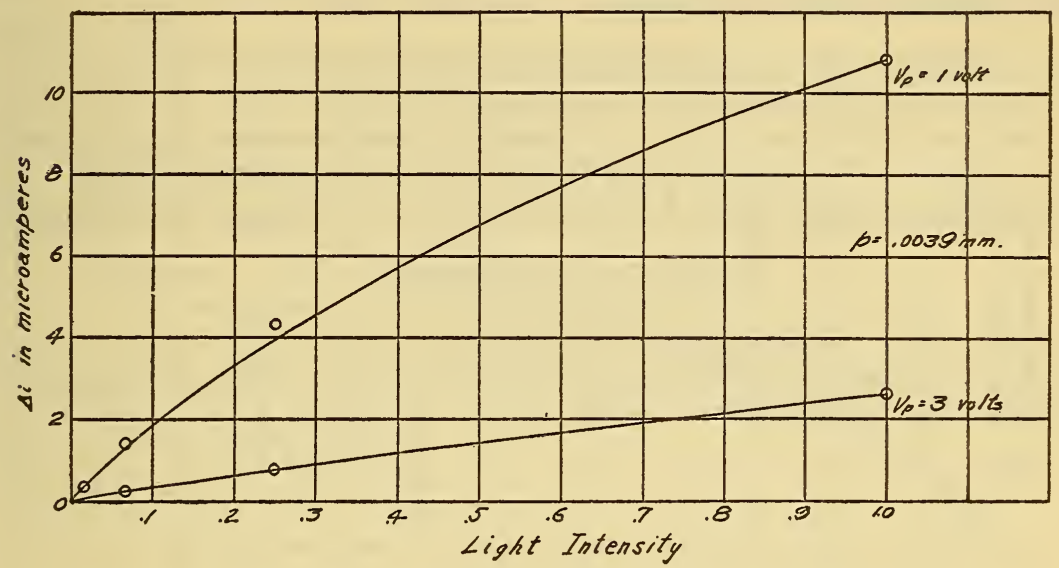

FIGURE 5.-Variation of $\Delta i$, with light intensity for conditions noted on curves

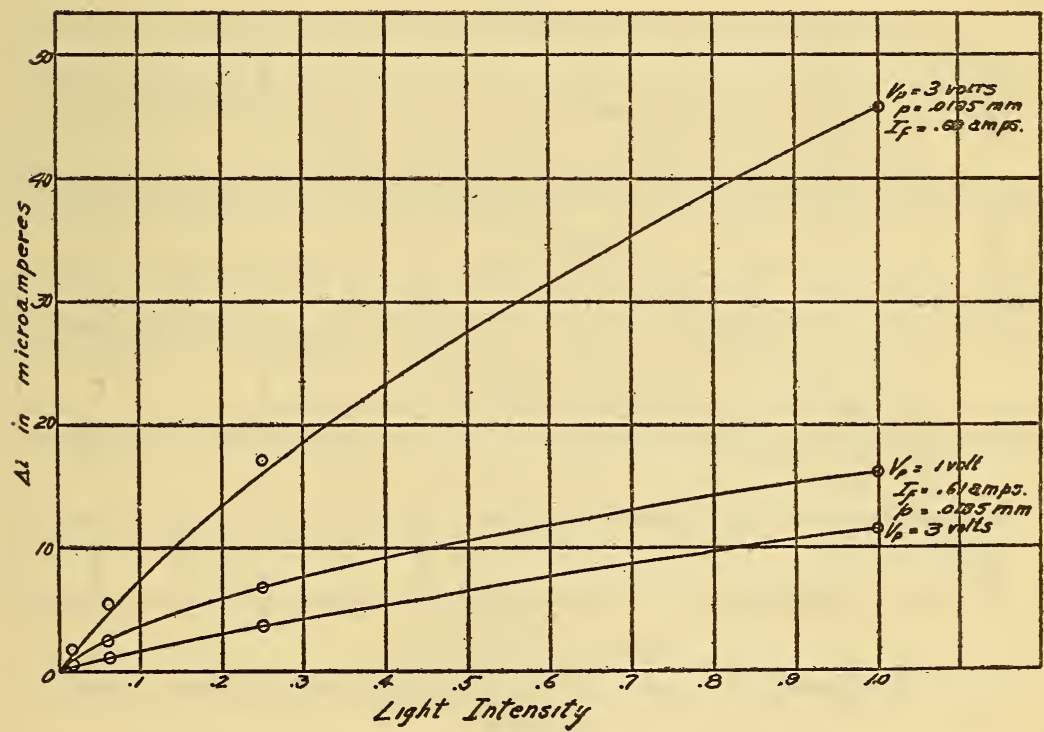

FIGURE 6.-Variation of $\Delta i$, with light intensity for conditions noted on curves

sidered justifiable because of the greater precision with which $\Delta i$ could be measured at this intensity. The curves shown in Figures $4,5,6,7$, and 8 are calculated from equation (3) using the values of $K_{3}$ obtained as outlined above, and given in Table 2 . 
The agreement as thus presented, is not striking, but as good as might reasonably be expected.

Inspection of the data shows a definite tendency for $K_{3}$ to decrease as applied voltage is increased. A definite tendency of $K_{3}$ to vary with the pressure is not so obvious.

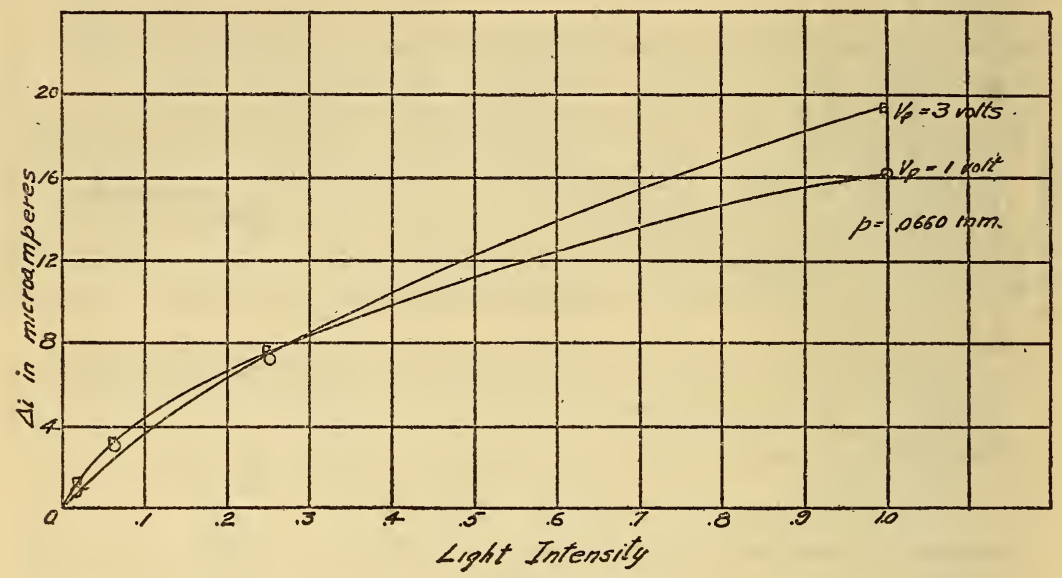

Figure 7.-Variation of $\Delta i$, with light intensity for conditions noted on curves

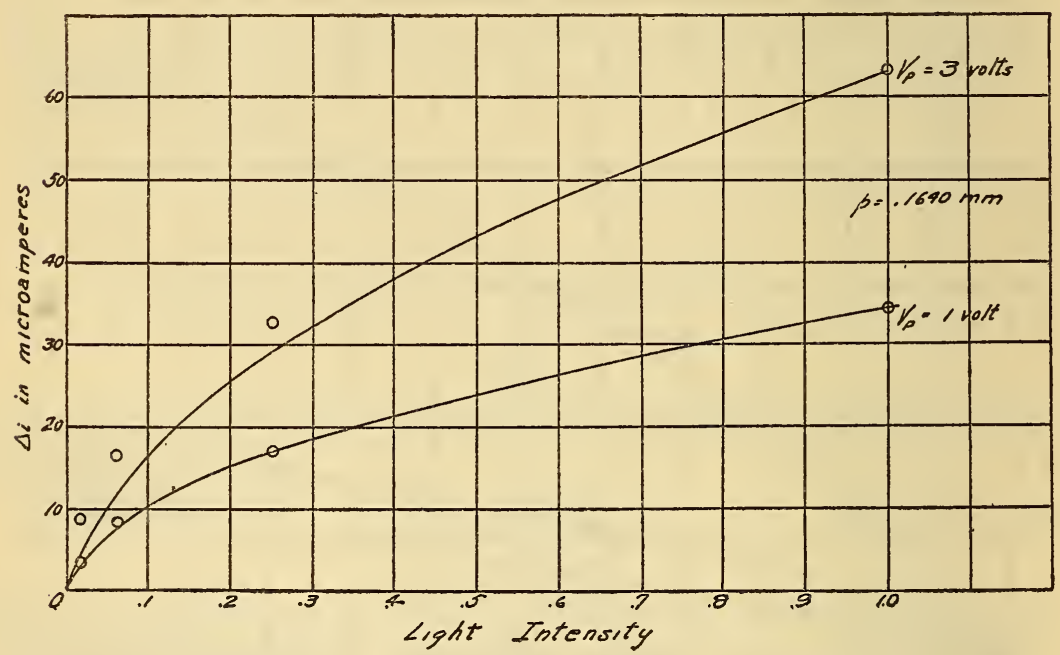

FIGURE 8.-Variation of $\Delta i$, with light intensity for conditions noted on curves

A loss of ions which will change with the electron current, can be pictured as a flow to the cathode. It would be difficult, however, to prove this loss proportional to the current. 


\section{CONCLUSIONS}

A tube was constructed in which the neutralization of space charge by positive ions was studied.

The positive ions were formed by the photo-electric ionization of caesium vapor.

The change in current due to the illumination was measured as a function of such controllable variables as pressure, light intensity, anode voltage, and cathode emission.

An empirical relation is given which by the choice of a single constant, may be made to fit the observations within the experimental error.

\section{ACKNOWLEDGMENTS}

The author wishes to acknowledge his indebtedness to Dr. F. L. Mohler under whose supervision this work was done. Besides placing the facilities of his laboratory at the author's disposal, he has offered many valuable suggestions.

W ASHington, June 1, 1929. 